\title{
Eruptive porokeratosis in an 80-year-old immunocompetent man
}

\section{Charlotte M. Smith ${ }^{1}$, Joseph Diehl' ${ }^{2}$ L. Evan Michael ${ }^{3}$, David Kent ${ }^{3}$}

\author{
${ }^{1}$ Srudent, Mercer University School of Medicine, Macon, GA, USA, ${ }^{2}$ Dermatologic Surgery, Mercer University School of \\ Medicine, Macon, GA, USA, ${ }^{3}$ Atlanta Dermatopathology, Atlanta, GA, USA
}

Corresponding author: Charlotte M. Smith, E-mail: charlotte.michelle.smith@live.mercer.edu

Sir,

Porokeratosis is typically an asymptomatic or mildly pruritic disorder of atrophic epidermal lesions surrounded by a hyperkeratotic border, histologically representing cornoid lamella. The most common clinically distinctive forms include classic porokeratosis of Mibelli, disseminated superficial porokeratosis (DSP), disseminated superficial actinic porokeratosis (DSAP), linear porokeratosis, dissiminated plantar and palmar porokeratosis, and punctate porokeratosis. Here we report a case of intensively pruritic, eruptive disseminated porokeratosis with acute onset, another subset that is rarely reported.

An 80-year-old immunocompetent Caucasian male with a several decade diagnosis of DSAP presented with pruritic, burning, painful, erythematous plaques located on the arms, hands, legs, and trunk. He was being treated for DSAP with doxepin but was still having intensely pruritic outbreaks of lesions twice a year that would last for 6 to 8 weeks and then dissipate. The lesions developed suddenly and acutely worsened. Brightly erythematous and edematous $5-10 \mathrm{~mm}$ atrophic scaly papules with elevated borders and a thin ribbon of scale involved all extremities and his chest (Figs. 1 and 2). The palms, soles, head, and mucosal surfaces were spared. Punch biopsies were taken and revealed parakeratotic columns with loss of the granular layer and presence of scattered necrotic keratinocytes, compatible with cornoid lamellae and suggestive of porokeratosis (Fig. 3). There was mild epidermal spongiosis with focal spongiotic microvesicle formation (Fig. 4). Within the dermis, there was a perivascular lymphohistiocytic inflammatory infiltrate with scattered eosinophils (Fig. 5). PAS stain was negative for fungal organisms. Histology and clinical symptoms suggested the diagnosis of eruptive disseminated porokeratosis. The patient was treated with methylprednisolone $5 \mathrm{mg}$ tablets in a dose pack and triamcinolone cream. His symptoms of intense pruritis were improved upon follow-up two weeks later. All lesions were healed except for a few remaining lesions on the legs.

DSAP is a variant of porokeratosis that presents with nonpruritic, nonpainful, scaly, erythematous papules/ plaques on sun-exposed skin areas. A rare subset of this disease is eruptive disseminated porokeratosis (EDP), which presents with acute onset of intensely pruritic erythematous papules [1]. EDP differs from DSAP in that it has an acute onset of plaques that are typically pruritic. DSAP develops over years and is typically

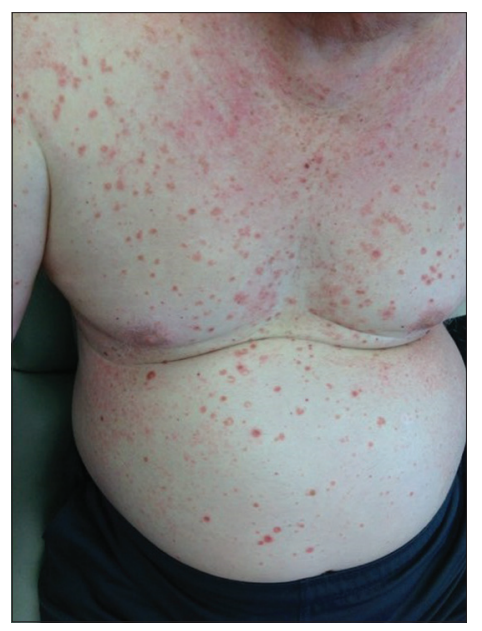

Figure 1: Brightly erythematous and edematous 5-10 mm atrophic scaly papules with elevated borders and a thin ribbon of scale on the chest and abdomen.

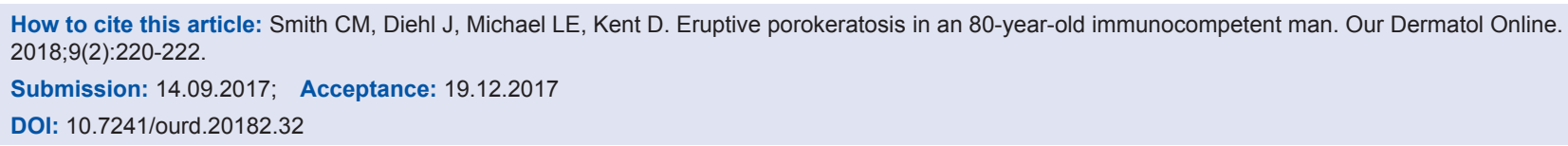




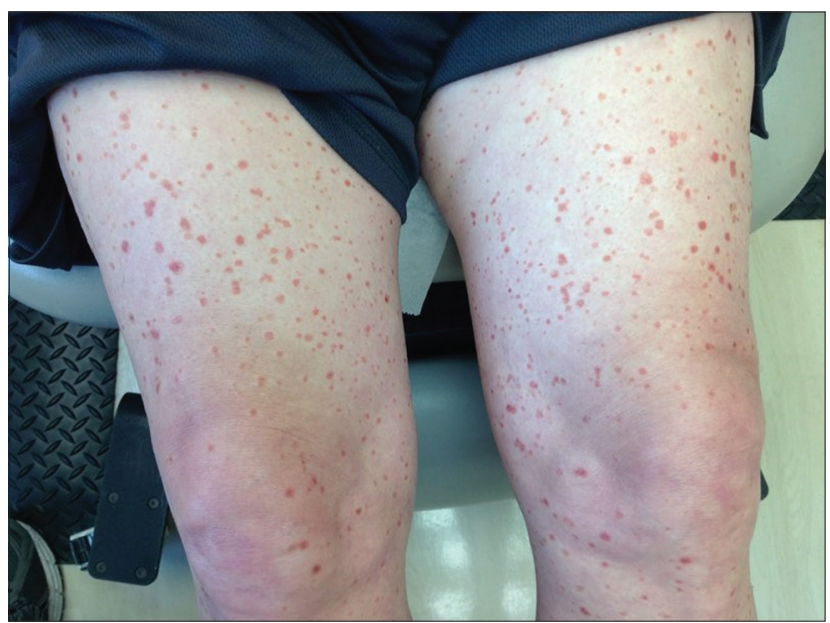

Figure 2: Brightly erythematous and edematous 5-10mm atrophic scaly papules with elevated borders and a thin ribbon of scale on bilateral legs.

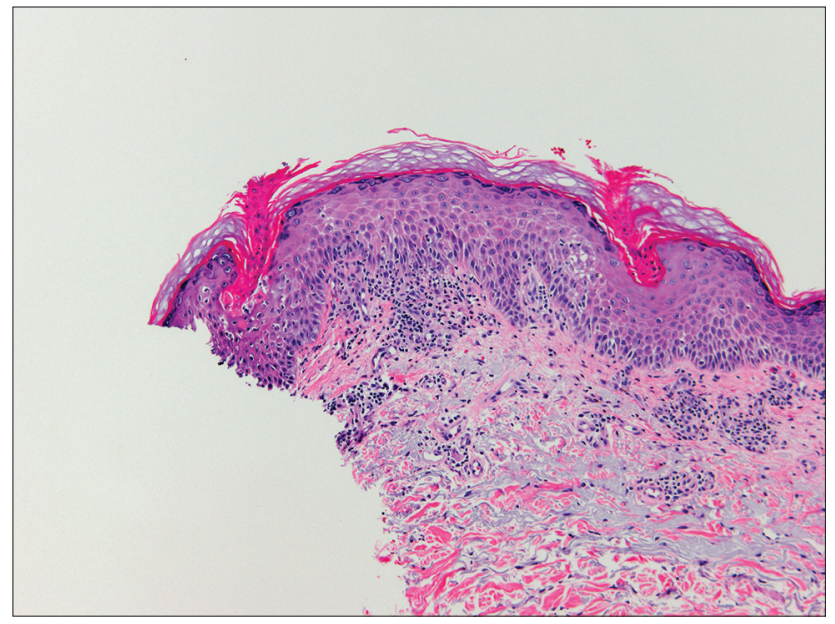

Figure 3: Angled columns of parakeratosis with loss of granular layer and necrotic keratinocytes in the subjacent epidermis, consistent with cornoid lamellae.

cosmetic with little or no symptoms. Only around 10 cases of EDP have been previously reported [2]. Histology typically demonstrates cornoid lamellae, epidermal spongiosis, and a perivascular inflammatory infiltrate of eosinophils and lymphocytes $[1,3]$. The course of EDP is similar to that of the presented patient, with repeated flares of intensly pruritic lesions and eventual regression. Our patient had hypertension but was an otherwise healthy 80-year-old man. Although initially diagnosed with DSAP, his clinical course and histologic findings are more consistent with the diagnosis of eruptive or inflammatory porokeratosis. Several terms have been proposed to describe this rare, atypical variant, including inflammatory DSP, eruptive pruritic papular porokeratosis, and eruptive disseminated porokeratosis [4]. It has been proposed that the inflammatory change leading to the intensely

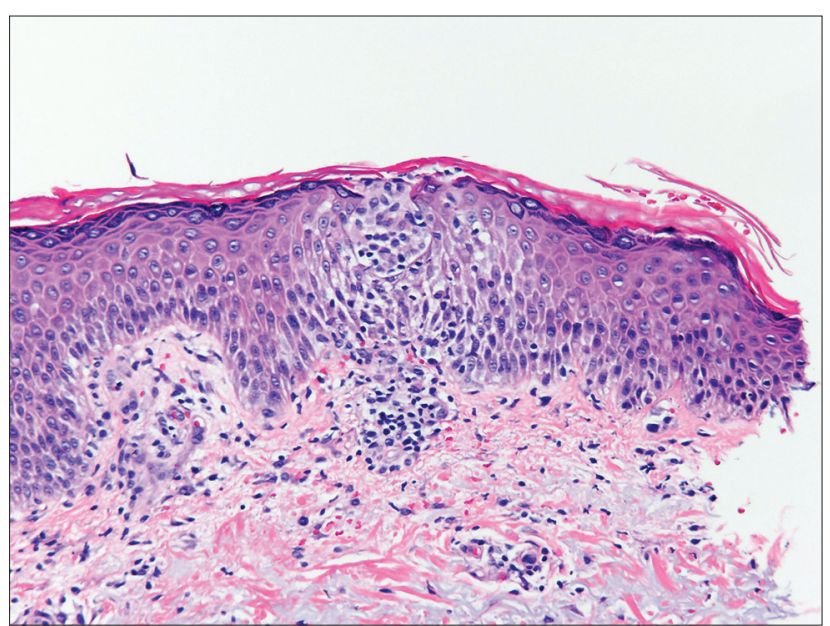

Figure 4: Epidermal spongiosis with spongiotic microvesicle formation.

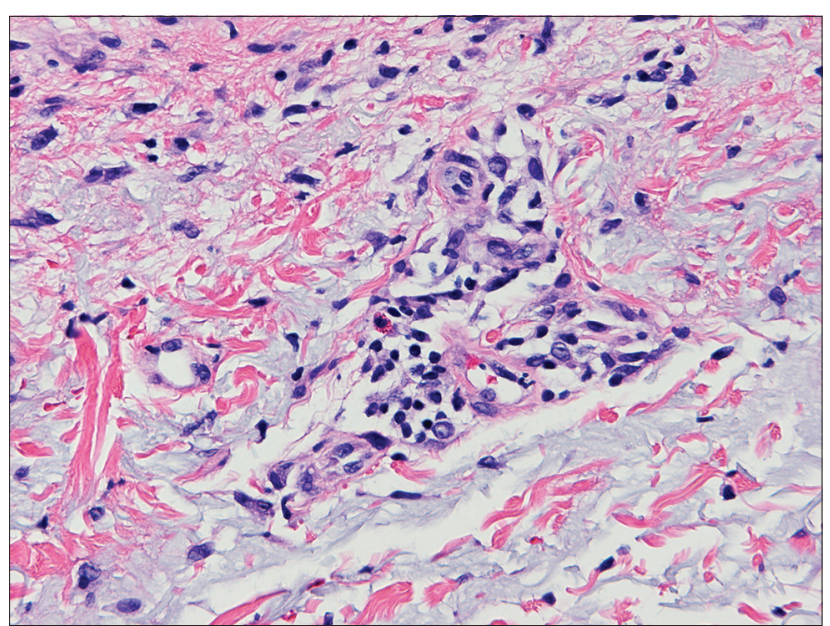

Figure 5: Perivascular inflammatory infiltrate with eosinophils.

prurutic erythematous papules may result from an immunological reaction against the abnormal clones of DSP, suggesting that porokeratosis is a precursor skin manifestation of malignant tumors [3,5]. Tanaka et al. postulated that regression of lesions was due to a CD4+ T cell-mediated immune reaction against the abnormal clones that resided in the epidermis [3]. Although the lesions typically resolve within several weeks, topical, intralesional and/or systemic steroid treatment results in improvement of symptoms. Use of topical 5-fluorouracil ointment has also been reported [1,3] Although EDP is considered a relatively rare disorder, it is imperative for dermatologists to be aware of this disease and consider it in their differential diagnosis.

\section{REFERENCES}

1. Kanzaki T, Miwa N, Kobayashi T, Ogawa S. Eruptive pruritic papular porokeratosis. J Dermatol. 1992;19:109-12. 
www.odermatol.com

2. Tee SI, Chong WS. Eruptive pruritic papular porokeratosis. Indian J Dermatol Venereol Leprol. 2012;78:758-60.

3. Tanaka M, Terui T, Kudo K, Tagami H. Inflammatory disseminated superficial porokeratosis followed by regression. Br J Dermatol. 1995;132:153-5.

4. Shoimer I, Robertson LH, Storwich G, Haber R. Eruptive disseminated porokeratosis: A new classification system. J Am Acad Dermatol. 2014;398-400.

5. Otsuka F, Shima A, Ishibashi Y. Porokeratosis has neoplastic clones in the epidermis: microflurometric analysis of DNA content of epidermal cell nuclei. J Invest Dermatol. 1989;92:231S-3S.

Copyright by Charlotte M. Smith, et al. This is an open-access article distributed under the terms of the Creative Commons Attribution License, which permits unrestricted use, distribution, and reproduction in any medium, provided the original author and source are credited.

Source of Support: Nil, Conflict of Interest: None declared. 